\title{
Analyzing the Learning Modes of Learners using Time-Management Modules in Self-Paced Learning
}

\author{
Juin-Ling Tseng, Member, IEEE and Ing-Chyi Pai
}

\begin{abstract}
Enhancing the effectiveness of e-learning is an important topic today. Many factors influence the effectiveness of learning, among which time management has the most direct impact on self-paced learning. This study developed a calendar time-management module to record the learning process in an self-paced learning environment. After analyzing the learning modes, we extracted learners that displayed intensive learning towards the end of a course period. We implemented two types of time management modules on the extracted subjects: a countdown timer and a course schedule module, and then analyzed the influence of the time modules on the learners in self-paced learning. The objective was to promote diligence by helping learners to begin learning earlier in the course period. Our results demonstrate that the incorporation of the countdown timer and course schedule time-management modules altered the distribution of study times and prompted all of the learners to complete the reading of course materials. The countdown timer module presented a stronger correlation with the tendencies of time management and the use of the time modules. This indicates that learners who are sensitive to changing numbers are more likely to follow a set course. Overall, the time modules differed in the degree of impact according to the characteristics of learners; however, the use of time modules was proven to enhance the effectiveness of studying.
\end{abstract}

Index Terms-Learner control, learning type, Self-Paced Learning, time-management modules

\section{INTRODUCTION}

$\mathrm{E}$ -LEARNING is a type of self-disciplined learning that (t) In teaching and learning, asynchronous teaching platform systems demonstrate two aspects: Learner Control and Program Control. In the former, learners pace their learning according to their own progress. However, different learners possess different learning motives and cognitive abilities, and for this

Manuscript received September 1, 2012. This work was supported by the National Science Council of the Republic of China, Taiwan, under Contract No. NSC100-2511-S-159-001.

Juin-Ling Tseng is with Department of Computer Science and Information Engineering, Minghsin University of Science and Technology, Hsin Feng, Hsin Chu County 30401, Taiwan. (phone: +886-3-559-3142; fax: +886-3-557-4321; e-mail: flysun@must.edu.tw)

Ing-Chyi Pai was with Graduate Institute of eLearning Technology, National Hsinchu University of Education, Hsin Feng, Hsin Chu County, Taiwan.(e-mail: chyi.pai@gmail.com) reason, many learners have difficulty deciding on the optimal learning mode [8, 13]. Clark and Mayer [7, 9] claimed that learners should utilize the settings of teaching systems to pace their learning for more effective self-regulated learning.

Time management has a stronger impact on learning in an online environment than in a classroom [1, 2]. Carroll [4] used the time required to learn things as a unit of measurement to establish a learning model. Klob [5] investigated the influence of environment on a learner's approach to time control. Roper conducted interviews on online learning strategies in which the importance of time management was discussed.

As shown in Fig.1, Hwang and Wang [10] examined the reading patterns in e-learning environments from the perspective of time and discussed the methods of completing online reading courses and the types of learning methods displayed. Based on learning time, they divided learners into four types: diligent learners, learners that display a posterior burst, learners that display a prior burst, and negligent learners. Among these types of learners, the learning effectiveness of diligent learners was the highest.

This study established an online learning system incorporating a countdown timer [3] and course progress time management module [14] in an attempt to alter the learning mode of learners that display a posterior burst, as shown in Fig.2. Our objective was to inspire them to begin studying

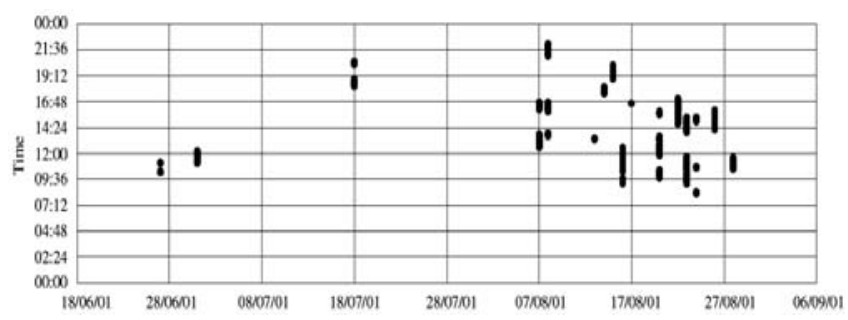

Fig. 1. A typical pattern of the Posterior Burst [10]

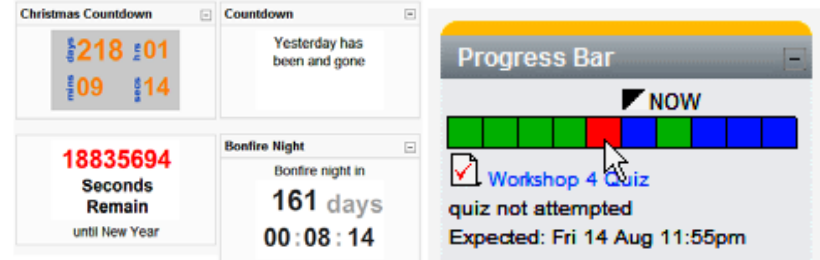

Fig. 2. Time Modules - Countdown timer[3] and Progress Bar [14] 
earlier in the course and adjust their learning process into that of a diligent learner, thereby increasing learning effectiveness.

\section{RELATED WORK}

\section{A. Self-Paced Learning}

E-learning requires that learners pace their learning by themselves [6]. In such a learning environment, the role of the learner switches from passive to active. The design of asynchronous online teaching platforms provides two types of learning mode:

1) Learner Control: This refers to the instruction methods available for different teaching events from among specific guidance models [12]. An online teaching system enables nonlinear control for a sequence of teaching themes. At the end of the sequence is program control. In contrast, the system provides multiple options for learning, which is Learner Control [11].

Learner control is the most significant feature of e-learning. E-learning allows the learner to choose the themes that they want; they control their own pace in learning and decide for themselves whether to skip certain lessons or portions of lessons. When users can make their own choices, we can say that this teaching program provides Learner Control.

2) Program Control: In self-paced learning models, environmental resources are a key factor providing the learner with additional options with regard to learning time and the order of the content. When only a few options are provided, most of which are learning plans from the designer, we refer to this as Program Control.

Due to the different learning motives and cognitive abilities displayed among learners, the majority of learners have difficulty selecting the optimal learning mode. Therefore, Program Control should be incorporated to assist learners in pacing their learning and achieving cognitive self-regulated learning.

\section{B. Types of learner behavior in e-learning}

To investigate the types of learner behavior in e-learning, Hwang and Wang examined the correlation between learning interaction, learning effectiveness, and learning time. Based on the distribution of study times in a course period, they defined four types of learners: diligent learners, learners that display a posterior burst, learners that display a prior burst, and negligent learners. From their learning achievements, they concluded that diligent learners were the best among the four types, followed by learners that display a posterior burst, learners that display a prior burst, and negligent learners. Hwang and Wang claimed that diligent learners often display a more positive side in interaction, often asking questions and assisting others. Learners that display a prior burst often express a sense of frustration and powerlessness; this type of learner often complains, criticizes, and is pessimistic. Negligent learners are more active in finding study partners and may take advantage of their interaction to obtain answers. Learners that display a posterior burst rarely interact with their teachers or peers. The primary subjects of this study are learners that display a posterior burst. In this study, we employed time management modules to provide learners that display a posterior burst with more stimulation during their learning process and steer them towards the diligent type of learning mode, thereby increasing their learning effectiveness.

\section{METHODOLOGY}

\section{A. Research Framework}

In order to identify learners that display a posterior burst and adjust their learning modes to that of diligent learners, we constructed a two-phase research framework to determine the relationships among the learning process of individual learners, the degree of course completion, and time modules as well as their influence on the time management of individuals.

1) Phase One: The primary objective of this phase was to record the online learning process of all the subjects and identify which type of learner they are. To achieve this, we constructed learning materials and a calendar time module in a Moodle environment to notify learners of their learning plans for the online courses and record their study times. In this phase, the learners were not divided into groups. Based on their learning processes, we identified learners that displayed a posterior burst for group testing in the second phase.

2) Phase Two: We randomly divided the learners that displayed a posterior burst into two groups: one with a countdown timer module and the other with a course schedule module. After the experiment, we performed assessments of their learning achievements. The focus of this phase was to analyze the changes that occurred in the learning processes of the subjects after the incorporation of the time modules and determine whether the approaches effectively enhanced their learning effectiveness.

\section{B. Time-Management Modules}

1) Calendar: A two-way mechanism in which teachers and learners could post messages. The messages posted by teachers were presented in green whereas those posted by the students were presented in blue. Teachers could remind learners of course deadlines and learners could use the module to plan their progress, as shown in Fig.3.

2) Countdown timer: A countdown to the deadline of each unit course. The purpose of this module was to convert deadlines on the calendar into a numerical figure that increased the time awareness of learners and reminded them to arrange their study times as time counted down. The time displayed referred to a deadline for a particular unit as suggested by the teacher, as shown in Fig.4.

3) Course schedule: a schedule on which learners could view their progress marked in any given unit. The progress suggested by teachers was also made available for the reference of learners in their arrangements, as shown in Fig.5.

$>$ The numbers in the little blocks in the schedule represent 
the quantity of teaching materials in the unit course.

$>$ When the cursor runs over the blocks, the learning themes of the unit are shown. Moreover, unread themes were marked as well as the scheduled time of completion.

$>$ Clicking the blocks provided access to the page of the teaching materials, on which major reminders were presented in three colors. Once a theme had been clicked and read, the corresponding block would turn green and be marked as already read; "current progress" indicated that the theme was scheduled to be read that day and themes that had not been read and the deadlines of which had already passed were highlighted in red and marked as unread. However, before the unit was closed completely, the learners could still read the materials; the purpose was to remind learners about the themes that they had missed. Themes that were scheduled to be read after the next day were highlighted in blue and also marked as unread. As long as the units were not closed, learners could exceed the standard schedule if they wished.

\section{Implementation process}

Because the subjects would be required to arrange their own study times at home or at school, we sought subjects that were familiar with computers and would be able to engage in

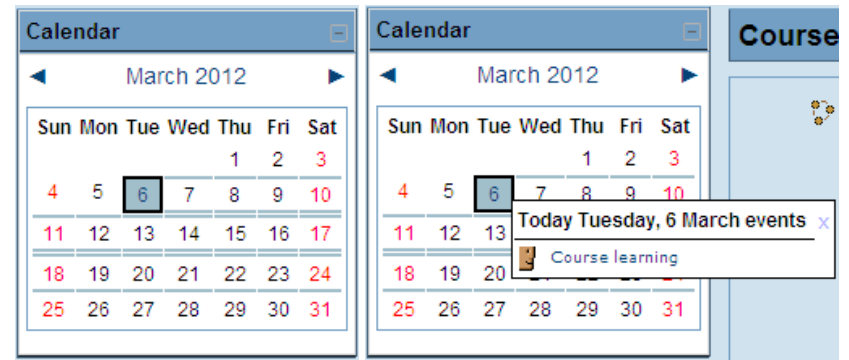

Fig. 3. The usage of calendar module

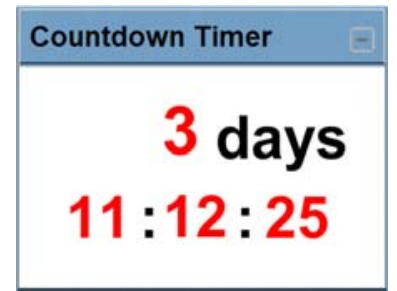

Fig. 4. The usage of countdown timer

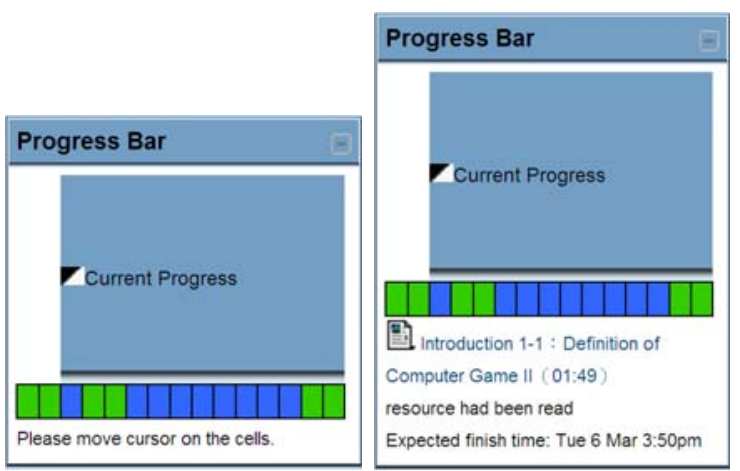

Fig. 5. The usage of progress bar autonomous learning. Thus, we enlisted college sophomores majoring in information engineering at a university of science and technology as the subjects of this study. The learning materials included an elective multi-media course.

During the first phase, we implemented the calendar time module with a basic introduction to digital game planning. During this phase, the learners engaged in autonomous learning. Following completion of the course, we conducted an assessment, and the subjects were required to fill out a questionnaire. Based on the definitions given by Hwang and Wang, we were able to identify learners that displayed a posterior burst for the second phase.

In the second phase, we evenly divided the learners that displayed a posterior burst into two groups and implemented the countdown timer and course schedule modules. The planned course was an intermediate course following the course in the first phase. The teacher incorporated the required learning times for each unit into the modules; the learners could refer to the time given by the system or study at their own pace. After completing the course in the second phased, we performed another assessment and administered a self-assessment questionnaire on the use of the Moodle time modules.

\section{RESULTS}

This study used Moodle as the primary online teaching platform with time modules. The main results of each phase are as follows:

\section{A. First phase: Using the calendar module to identify learners that displayed a posterior burst}

The basic introduction to games in the first phase included a total of 6 units with links to 74 pages of materials. The total duration of the course was approximately 114 minutes, and the learning deadline was 18 days. Each page in the units displayed calendar Moodle time modules with the learning deadline in a frame. A total of 69 learners participated in this phase of the study, reading the course material according to the study plan, taking the online assessment, and completing the self-assessment questionnaire.

We created a learning distribution graph using the number of times the learners logged in each day. The curve connecting the rhombic dots shows the distribution of the study times displayed by learners in the first phase. We then created a trend curve for the number of study times with every 2 days as a unit. As shown in Fig.6, the entire experiment population exhibited the characteristics of learners that display a posterior burst.

Based on statistics regarding the distribution of study times in the first phase, we determined that none of the experiment population were diligent learners or learners that displayed a prior burst. Fifty learners displayed a posterior burst and 19 were negligent learners. This shows that the majority of the learners demonstrated passive attitudes towards online learning. The 50 learners that displayed a posterior burst were randomly divided into two groups (25 learners in each group). The 


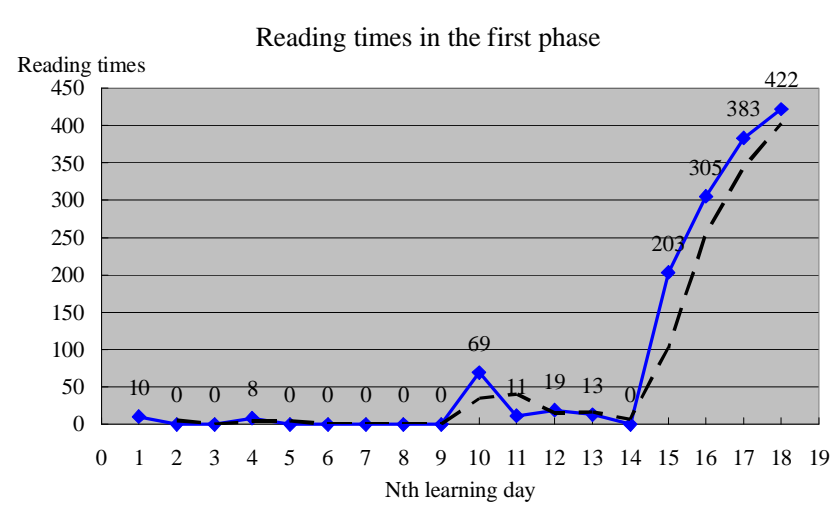

Fig. 6. Reading times for each day in the first phase

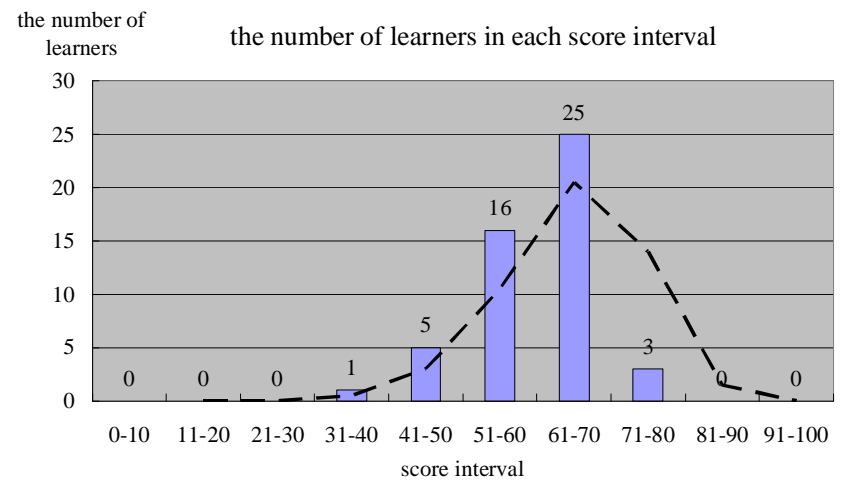

Fig. 7. The number of learners in each score interval in the first phase

countdown timer and course schedule time modules were then respectively implemented in the two groups to examine the influence of the two types of time module on learners that display a posterior burst.

The study time records of individual learners and the entire subject population presented a total of 1,443 reads. If we divide this number by the total number of pages in the materials (74), we can see that the 50 learners that displayed a posterior burst contributed to an average of only 20 reads per page in the first phase. If all 50 learners had completed the full reading course, they should have contributed to 50 reads for each page. This shows that the entire subject population of learners that displayed a posterior burst completed only $40 \%$ of the entire course. In other words, most of the subjects did not read or even access all of the course materials. In learning achievement, the average score obtained on the assessment test in the first phase was 60.07; over half of the subjects did not achieve the standard assessment score of 70, as shown in Fig.7.

\section{B. Group 1 in the second phase: influence of countdown timer module on learners that displayed a posterior burst}

The intermediate game planning course in the second phase included 4 units with links to 57 pages of materials. The total duration of the course was approximately 80 minutes, and the learning deadline was 16 days. In this phase, a countdown timer module was implemented in one of the two experimental groups with 25 learners that displayed a posterior burst.

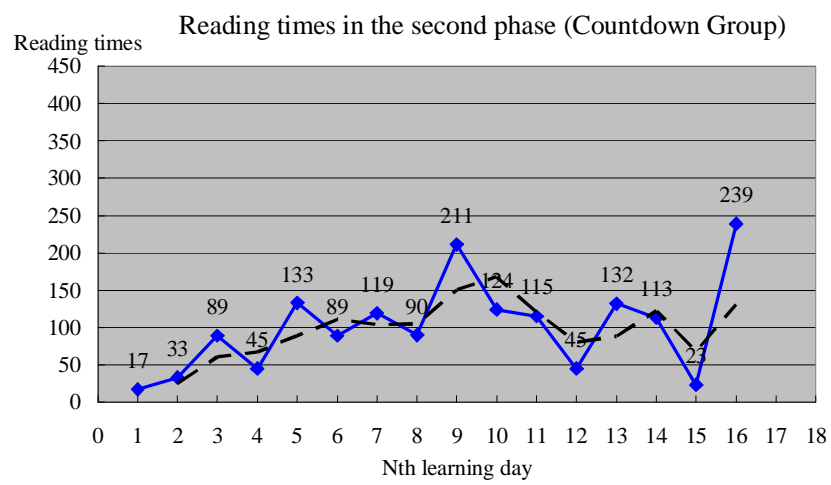

Fig. 8. Reading times of countdown group for each day in the second phase

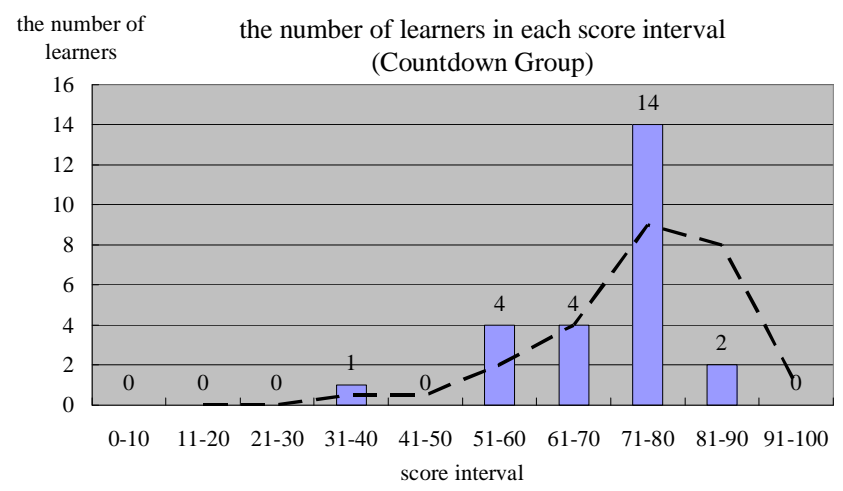

Fig. 9. The number of learners in each score interval in the second phase

Similarly, the subjects were required to read the materials online, take the online assessment test, and complete the self-assessment questionnaire.

The daily statistics of the learners in this group are exhibited in Fig.8. Clearly, the learners began reading the materials in the first part of the total study duration, and portions of the learners continued reading until the learning deadline.

To analyze the influence of the countdown timer module on learners that display a posterior burst, we compared the study time curves of the calendar module in the first phase and the countdown timer module in the first phase. Our results revealed a concentration of reading near the end of the learning period in the first phase; however, the incorporation of the countdown timer led to a more even distribution of reading times throughout the second learning period. In other words, the countdown timer adjusted the behavior of learners that displayed a posterior burst to one more similar to that of diligent learners.

In terms of completion, participants accumulated a total of 1,617 reads in the second phase. Divided by the number of pages in the materials (57), we derived an average of 28 reads for each page (more than the 25 reads if each of the 25 learners had only read each page once). Furthermore, based on the study records, all of the learners accessed each unit at least once. In other words, we can infer that the learners in this group read all of the course materials at least once, and some of the learners reread some of the materials. In learning achievement, 16 of the 


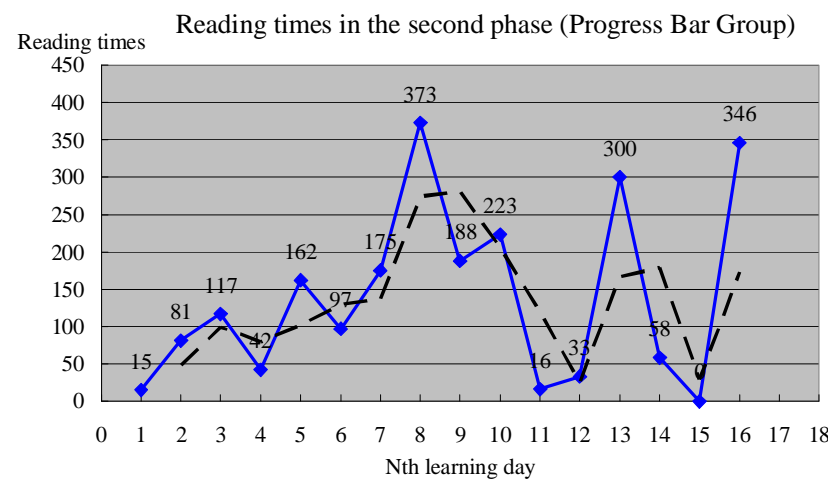

Fig. 10. Reading times of countdown group for each day in the second phase

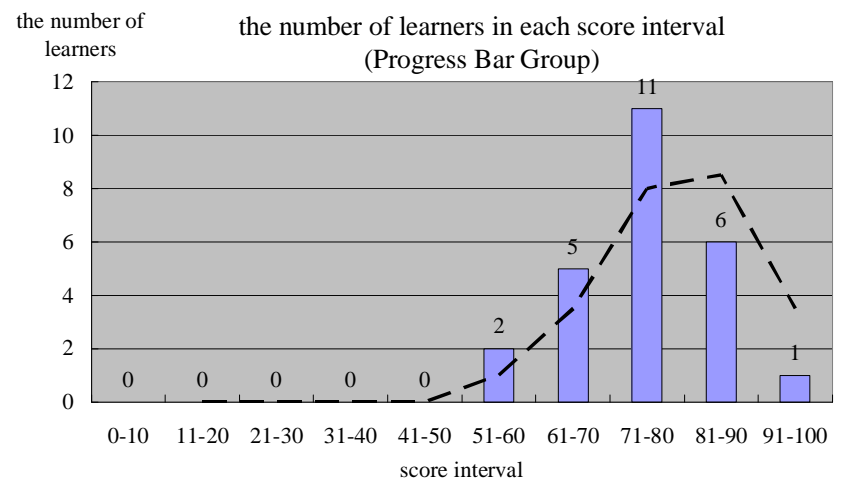

Fig. 11. The number of learners in each score interval in the second phase

25 learners (more than half) achieved the standard score of 70 for course certification, as shown in Fig.9.

C. Group 2 in the second phase: influence of course schedule module on learners that displayed a posterior burst

In this phase, this group was placed in a similar learning environment as Group 1; however, a course schedule was used instead of the countdown timer. The subjects in this phase accumulated a total of 2,226 reads, accounting for an average of 39 reads for each of the 57 pages (more than the 25 reads if each of the 25 learners had only read each page once), as shown in Fig.10. Furthermore, based on the study records, all of the learners accessed each unit at least once. In other words, we can infer that the learners in this group read all of the course materials at least once, and the majority of the learners reread some of the materials. In learning achievement, 18 of the 25 learners (more than half) achieved the standard score of 70 for course certification, as shown in Fig.11.

\section{CONCLUSION}

This study explored methods to increase the completion rate of online course reading from the perspective of time. We designed Moodle time modules to assist learners in autonomous learning. To observe how learners make use of the learning period to read online materials, we did not restrict access to the materials (after a given period) to control study times. In the first phase of this study, we identified learners that displayed a posterior burst from our subject population. To improve the learning behavior of these learners, we incorporated a countdown timer and course schedule in the second phase of our study. Our experiment results demonstrate that the incorporation of the countdown timer and course schedule modules effectively stimulated these learners to be more active in learning. The learners increased the number of times they read the materials and displayed better time management, which further improved their learning effectiveness. From the aspects of time management and the use of time modules, we discovered that learners displayed traditional passive study patterns when using the reminders in the calendar module. The course schedule and countdown timer modules assisted the teacher to instill better learning plans and improved their effectiveness in learning. Teachers could make use of course schedules and countdown timers in future online learning systems to inspire learning and enhance learning effectiveness in learners.

\section{REFERENCES}

[1] L. W. Anderson, Perspectives on school learning: Selected writings of John B. Carroll, Taylor \& Francis, 1985.

[2] D. C. Berliner, What's All the Fuss about Instructional Time?, The Nature of Time in Schools. NY: Teachers College Press, 1990.

[3] M. Cannings, F. Liauw and C. Owen, Countdown Block (Version 2.0): Moodle, 2008.

[4] J. B. Carroll, “A model of school learning," Teachers College Record, vol.64, no.8, 723-733, 1963

[5] D. A. Kolb, "Experiential Learning Theory: Previous Research and New Directions," Perspectives on Thinking, Learning, and Congnitive Styles pp.227-247, 2001.

[6] B. J. C. Claessens, "Perceived Control of Time," Time Management and Personal Effectiveness at Work: Technische Universiteit Eindhoven, 2004.

[7] R. C. Clark and R. E. Mayer, e-Learning and the Science of Instruction:Proven Guidelines for Consumers and Designers of Multimedia Learning. CA: Pfeiffer, 2003.

[8] K. Ely, T. Sitzmann and C. Falkiewicz, "The influence of goal orientation dimensions on time to train in a self-paced training environment," Learning and Individual Differences, vol.19, pp.146-150, 2009.

[9] R. D. Hannafin and H. J. Sullivan, "Learner control in full and lean CAI programs,” Educational Technology Research and Development, vol.43, no.1, 19-30, 1995.

[10] W.-Y. Hwang and C.-Y. Wang ,"A study of learning time patterns in asynchronous learning environments," Computer Assisted learning, vol.20, no.4, 292-304, 2004

[11] B.Lin and C.T. Hsieh, "Web-based teaching and learner control: a research review," Computers \& Education, 37(3-4), 377-386, 2001.

[12] M. D. Merrill, "What is Learner Control?” (No. ERIC \#ED 298-905), 1984.

[13] P. R. Pintrich, D. A. F. Smith, T. Garcia and W. J. McKeachie, “A Manual for the Use of the Motivated Strategies for Learning Questionnaire (MSLQ)," (No. ERIC \#ED338122 ): National Center for Research to Improve Postsecondary Teaching and Learning, Ann Arbor, MI, 1991.

[14] M. d. Raadt, Progress Bar [Block]: Moodle, 2009.

Juin-Ling Tseng (M’01) became a Member (M) of IEEE in 2001. He received his B.S. from SooChow University in 1994 and M.S./Ph.D. from Chung Yuan Christian University, Taiwan, in 1996/2006. Currently, he is an assistant professor in the Dept. of Computer Science and Information Engineering at the 
Minghsin University of Science and Technology, Taiwan. His research interests include the areas of computer graphics and e-learning.

Ing-Chyi Pai received her M.S. from Graduate Institute of eLearning Technology, National Hsinchu University of Education in 2010. Her research interests include the areas of e-learning. 